\title{
A novel TSHR gene mutation (Ile691Phe) in a Chinese family causing autosomal dominant non-autoimmune hyperthyroidism
}

\author{
Zheng Liu · Yuanming Sun · Qingming Dong • \\ Mingliang He • Christopher H. K. Cheng • \\ Feiyue Fan
}

Received: 1 December 2007/ Accepted: 10 January 2008/Published online: 29 February 2008

(C) The Japan Society of Human Genetics and Springer 2008

\begin{abstract}
Familial non-autoimmune hyperthyroidism is a rare autosomal dominant genetic disease resulting from activating mutations in the thyroid-stimulating hormone receptor (TSHR) gene. In this work a Chinese family with autosomal dominant non-autoimmune hyperthyroidism across four generations was collected. The strongest evidence for linkage in this study occurred on chromosome 14q24.2-31.3. By mutation scan of the TSHR gene located within the region of interest, a heterozygote substitution $(\mathrm{A}>\mathrm{T})$ at position 2071 of the TSHR was found, changing isoleucine 691 to phenylalanine. Our study identified the first germline mutation in the intracellular C-terminal domain of TSHR.
\end{abstract}

Keywords TSH receptor .

Autosomal dominant non-autoimmune hyperthyroidism $(\mathrm{ADNAH}) \cdot$ Linkage analysis

Zheng Liu and Yuanming Sun contribute to this work equally.

Z. Liu $\cdot$ C. H. K. Cheng

Department of Biochemistry, The Chinese University

of Hong Kong, Shatin, NT, Hong Kong

Z. Liu · Y. Sun · F. Fan $(\square)$

Tianjin Key Laboratory of Molecular Nuclear Medicine, Institute of Radiation Medicine, Chinese Academy of Medical Sciences and Peking Union Medical College,

300192 Tian Jin, China

e-mail: fangao6@gmail.com

Z. Liu · Q. Dong · M. He

Stanley Ho Centre for Emerging Infectious Diseases

and $\mathrm{Li} \mathrm{Ka}$ Shing Institute of Health Sciences,

The Chinese University of Hong Kong, Shatin, NT, Hong Kong

\section{Introduction}

The thyroid-stimulating hormone receptor (TSHR) is a glycoprotein hormone receptor, belonging to the subfamily of seven transmembrane receptors, $G$ protein-coupled glycoproteins, and is the largest of all known glycoprotein hormone receptors (Nishi et al. 2000). TSHR has long been known to play a pivotal role in regulating thyroid gland physiology. It is involved in the late stages of thyroid organogenesis and is the major stimulator of thyroid cell growth, differentiation, and function (Grasberger et al. 2007). Naturally occurring TSHR mutations have been identified as one of the molecular causes of ADNAH (Duprez et al. 1994). In this study we report the first activating TSHR mutation located in the C-terminal domain of TSHR identified in a Chinese family affected with ADNAH.

\section{Materials and methods}

Subjects

A four-generation Chinese family with ADNAH was collected from the Institute of Radiation Medicine, Chinese Academy of Medical Sciences and Peking Union Medical College (CAMS and PUMC), located in Tian Jin, China (Fig. 1a). This study was approved by the Ethics Committee of the institute. All individuals in this family were invited to participate and gave informed consent to the study protocol. The study was conducted according to the laws of the declaration of Helsinki. A complete family history was obtained and 26 individuals from the family were sampled for clinical diagnosis with informed written consent. TSH, T3, T4, free $\mathrm{T} 4$, and free $\mathrm{T} 3$ were 
Fig. 1 Pedigree of the investigated family with ADNAH due to activating TSHR mutation I691F. a This family included 30 individuals. The proband is indicated by the arrow. Males are indicated by squares, females are indicated by circles. Black symbols represent affected individuals. b Results from direct sequencing of PCR-amplified genomic DNA from the Chinese family revealed a heterozygous mutation $2071 \mathrm{~A}>\mathrm{T}$. It demonstrated ATT $\rightarrow$ TTT mutation, affecting the isoleucine residue at position 691, which was replaced by a phenylalanine in the intracellular C terminus of TSHR protein
A
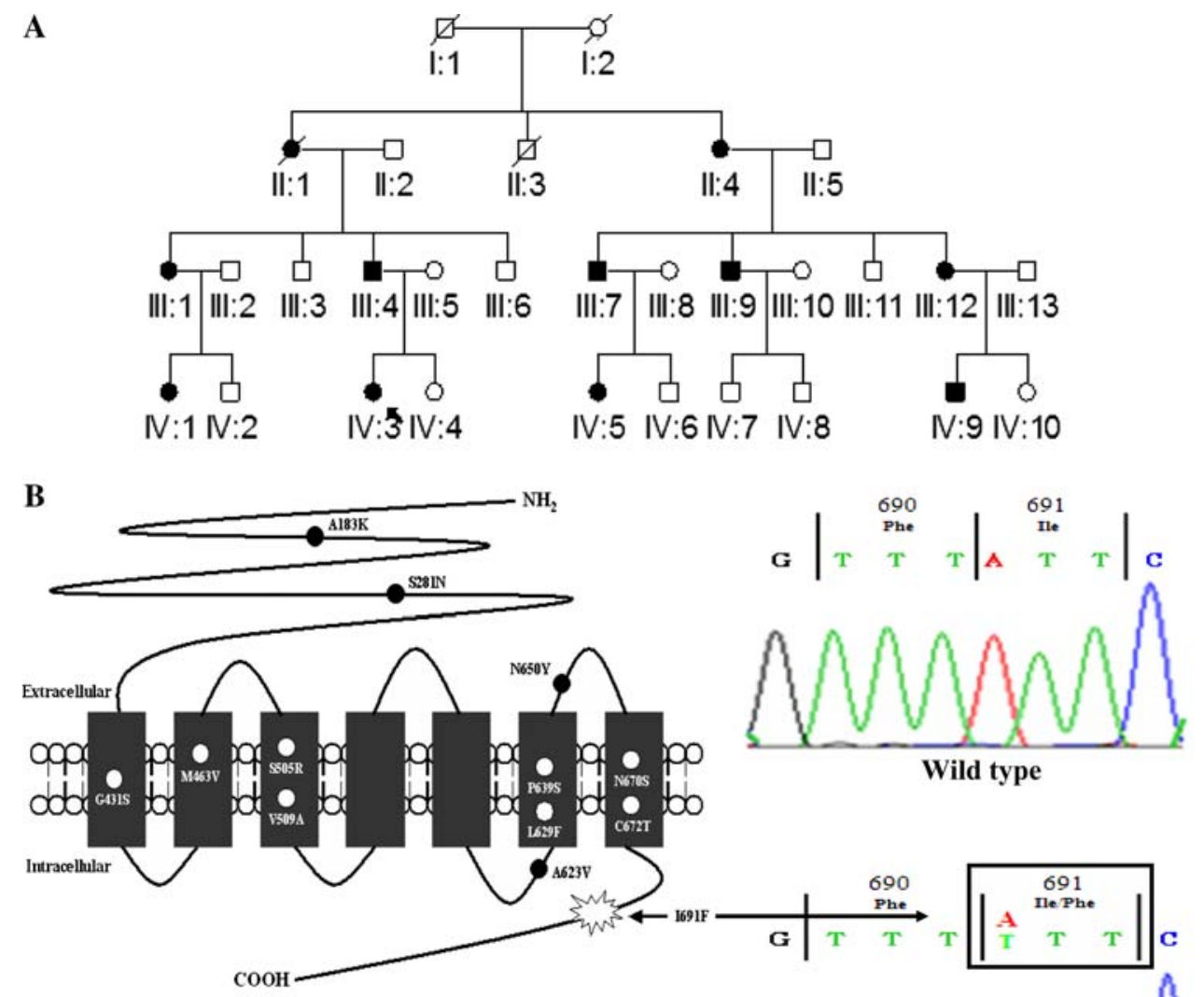

Wild type

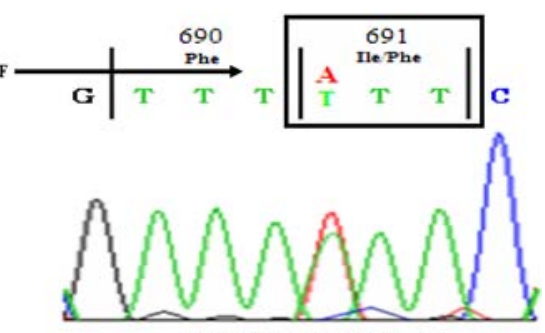

Mutation 2071 A $>$ T determined by electrochemiluminescent immunoassays (Roche Pharma, Switzerland) (Table 1). All 26 individuals from the family were sampled for genotyping and linkage analysis. Fifty normal individuals with no personal or family history of cancer were sampled as controls.

DNA extraction, genotyping, and linkage analysis

Genomic DNA was extracted from peripheral blood by use of a DNA extraction kit (TaKaRa, Japan) according to the manufacturer's instructions for genotyping and linkage analysis. Genome-wide screening was performed with 382 markers spaced an average of $10 \mathrm{cM}$ apart (ABI Prism Linkage Mapping Set, Version 2.5, Applied Biosystems, USA). Multiplex PCR was carried out in a $5 \mu$ reaction mixture as previously documented (Yang et al. 2006). Alleles were analyzed by Genescan analysis software v3.7 and Genotyper v3.7 (Applied Biosystems, USA). The MLINK program of the LINKAGE package (Version 5.1) was used for the calculation of two-point LOD scores. The disease was assumed to be an autosomal dominant trait
Table 1 Hormonal data of the patients

\begin{tabular}{llcrlrl}
\hline & $\begin{array}{l}\text { Age of } \\
\text { the patient } \\
\text { (year) }\end{array}$ & $\begin{array}{l}\text { Serum } \\
\text { TSH } \\
(\mathrm{mU} / \mathrm{l})\end{array}$ & $\mathrm{T} 3$ & $\mathrm{~T} 4$ & $\mathrm{FT} 3$ & $\mathrm{FT}$ \\
& $(\mathrm{ng} / \mathrm{dl})$ & $(\mathrm{ug} / \mathrm{dl})$ & $(\mathrm{ng} / \mathrm{dl})$ & (ng/dl) \\
\hline II:4 & 57 & $<0.1$ & 427 & 16.7 & 10.7 & 2.1 \\
III:1 & 38 & 0.14 & 312 & 15.2 & 9.7 & 1.8 \\
III:4 & 32 & $<0.1$ & 392 & 21.4 & 8.5 & 5.9 \\
III:7 & 32 & $<0.1$ & 267 & 18.5 & 6.4 & 4.2 \\
III:9 & 29 & 0.11 & 365 & 15.9 & 11.2 & 3.3 \\
III:12 & 26 & 0.18 & 489 & 20.4 & 4.3 & 7.2 \\
IV:1 & 10 & 0.26 & 239 & 22.3 & 5.7 & 2.4 \\
IV:3 & 8 & $<0.1$ & 502 & 16.5 & 9.6 & 3.5 \\
IV:5 & 7 & $<0.1$ & 376 & 16.9 & 8.5 & 4.6 \\
IV:9 & 3 & 0.12 & 367 & 14.1 & 7.9 & 3.4 \\
Normal & range & $0.3-4$ & $70-200$ & $4.2-11$ & $1.4-4.4$ & $0.7-1.6$ \\
\hline
\end{tabular}

with 99\% penetrance. Marker allele frequencies were set at $1 / n$, where $n$ is the number of alleles observed. We assumed gene frequencies of 0.0001 and no sex difference in recombination rates. 
Mutation detection in the family

The genes TSHR associated with ADNAH have been mapped within the region of interest. To identify the disease-causing gene, exons 1-10 of the TSHR gene were amplified with primers previously described (de Roux et al. 1996). In brief, PCR amplification was designed to produce five overlapping fragments that cover the whole length of exon 10. Exons 1 through 9 were amplified individually using nine pairs of intronic primers. The $100 \mu \mathrm{l} \mathrm{PCR}$ final volume contained $50 \mathrm{ng}$ genomic DNA and $1 \mathrm{U}$ Taq DNA polymerase (TaKaRa, Japan). The PCR products were sequenced by ABI 3100 automatic sequencer according to the manufacturer's recommendations and the PCR primers as sequencing primers.

\section{Results}

Characteristic of the patients

The four-generation Chinese family from Tian Jin city was identified and ten individuals were diagnosed with ADNAH by the Institute of Radiation Medicine, CAMS and PUMC. This family included 30 individuals; among them were four living affected males and six living affected females. Some affected individuals in this family recalled that they had been diagnosed as ADNAH at less than five years old (III:4, III:12, IV:3, IV:5, and IV:9). The proband of this family is an 8-year-old girl (IV:3) diagnosed as having non-autoimmune hyperthyroidism at two years of age. She had been experiencing sleep difficulties, hyperactive behavior, and irritability, but no temperature intolerance or bowel movement disturbances were observed.

Linkage analysis and germline TSHR mutation

By genome-wide scan and linkage analysis a maximum two-point LOD score of 5.76 at $\theta=0.1$ was found at D14S74 in this family, whereas two-point LOD scores were very low at other previously reported loci (Table 2). The strongest evidence of linkage was detected with three adjacent microsatellite markers genotyped between D14S258 and D14S68 on chromosome 14q24.2-31.3.

Mutation analysis of the TSHR gene detected no mutations in exons 1 through 9, but a single nucleotide change $(\mathrm{A}>\mathrm{T})$ in exon 10 at position 2071 of the TSHR was found in all affected members. This $2071 \mathrm{~A}>\mathrm{T}$ mutation leads to the replacement of an isoleucine (Ile, I) by a phenylalanine (Phe, F) at codon 691 in this Chinese family (Fig. 1b). The same mutation was not detected in 16
Table 2 Two-point lod scores between the causative gene and 9 markers of chromosome 14

\begin{tabular}{lrrrrrr}
\hline \multicolumn{7}{c}{ LOD score at $\theta$} \\
\cline { 2 - 7 } & \multicolumn{1}{l}{0.0} & \multicolumn{1}{l}{0.1} & \multicolumn{1}{l}{0.2} & \multicolumn{1}{l}{0.3} & \multicolumn{1}{c}{0.4} & 0.5 \\
\hline D14S261 & -17.26 & -4.98 & -2.22 & -1.09 & -0.20 & 0.00 \\
D14S275 & -13.20 & -3.55 & -1.79 & -1.02 & -0.12 & 0.00 \\
D14S288 & -25.87 & -9.47 & -4.54 & -2.07 & -0.87 & 0.00 \\
D14S63 & -9.65 & -1.19 & -1.01 & -0.89 & -0.06 & 0.00 \\
D14S258 & -2.03 & 3.79 & 1.07 & 0.61 & 0.04 & 0.00 \\
D14S74 & -1.93 & 5.76 & 4.79 & 2.14 & 0.78 & 0.00 \\
D14S68 & -7.67 & 1.07 & 1.69 & 1.02 & 0.60 & 0.00 \\
D14S280 & -13.22 & -3.69 & -1.83 & -1.07 & -0.14 & 0.00 \\
D14S985 & -16.43 & -4.29 & -1.98 & -0.97 & -0.17 & 0.00 \\
\hline
\end{tabular}

unaffected members and 50 controls (data not shown). The mutation was shown to co-segregate with the ADNAH phenotype, suggesting it was the disease-causing mutation in the family.

\section{Discussion}

So far, 27 activating point mutations of the TSHR have been reported by the TSHR database (http://innere. uniklinikum-leipzig.de/tsh). However, of these, only 12-point mutations can cause familial non-autoimmune hyperthyroidism. Since 1994, when Duprez first discovered the Val509Ala and Cys672Tyr mutations of TSHR in an ADNAH family, ten other constitutively active germline TSHR mutations have been reported, bringing the total to 12 (Duprez et al. 1994). As Fig. 1b shows, these mutations are all located within the extracellular region (A183K and $\mathrm{S} 281 \mathrm{~N}$ ), the transmembrane domain (G431S, M463V, S505R, V509A, L629F, P639S, N670S and C672T), or the loop region (A623V and $\mathrm{N} 650 \mathrm{Y})$, whereas mutation $\mathrm{I} 691 \mathrm{~F}$ is located in the intracellular C-terminal region.

In general, the TSH, after binding to its membrane receptor, the extracellular region of TSHR, switches the intracellular loop of the TSHR that interacts with the Gsalpha protein, then activates the cAMP pathway (Park et al. 2005). Site-directed mutagenesis experiments showed that the mutations in the extracellular region (A183K and S281N) increased the sensitivity to TSH (Smits et al. 2002; Neumann et al. 2005). This suggests these mutations in the extracellular region of TSHR and TSH do cooperate in the building of a novel module capable of constitutive activation of the cAMP pathway. However, experimental evidence indicated that mutation within the extracellular loop (N650Y) had a major effect on the conformation of the inactive receptor. The "hot spot" region for these 
mutations is localized in the transmembrane segments. Like the extracellular loop, molecular modeling indicates it has a pivotal role in intramolecular signal transduction and constrains the inactive TSHR state (Farid et al. 2000). This regional mutation allows direct contact of this domain with Gsalpha protein; it is most likely the intracellular loops mutation (A623V) that induces cAMP accumulation (Parma et al. 1993; Van et al. 1995).

Here, we report the first family with a constitutively active TSHR mutation in the C-terminal region resulting in a substitution of the conserved $\mathrm{Il}^{691}$ for Phe. Mutation I691F located in the C-terminal region causes the TSHR structural change. It can also affect the interaction between TSHR and Gsalpha protein. This suggests that the intracellular C terminus of the human TSHR plays an important role in receptor activation. In conclusion, this is the first time a mutation in the intracellular C-terminal region of the human TSHR has been identified, indicating that the mutations can be distributed over the entire intracellular region. It also provides important structural information about receptor activation and may aid better understanding of the structure-function relationship between the TSHR and Gsalpha protein.

Acknowledgments We would like to thank the family who participated in this study. This work was supported by a Grant from the National Nature Science Foundation of China (No. 30400240).

\section{References}

de Roux N, Misrahi M, Chatelain N, Gross B, Milgrom E (1996) Microsatellites and PCR primers for genetic studies and genomic sequencing of the human TSH receptor gene. Mol Cell Endocrinol 117:253-256
Duprez L, Parma J, Van Sande J, Allgeier A, Leclere J, Schvartz C, Delisle MJ, Decoulx M, Orgiazzi J, Dumont J (1994) Germline mutations in the thyrotropin receptor gene cause non-autoimmune autosomal dominant hyperthyroidism. Nat Genet 7:396401

Farid NR, Kascur V, Balazs C (2000) The human thyrotropin receptor is highly mutable: a review of gain-of-function mutations. Eur $\mathbf{J}$ Endocrinol 143:25-30

Grasberger H, Van Sande J, Hag-Dahood Mahameed A, TenenbaumRakover Y, Refetoff S (2007) A familial thyrotropin (TSH) receptor mutation provides in vivo evidence that the inositol phosphates $/ \mathrm{Ca}^{2+}$ cascade mediates TSH action on thyroid hormone synthesis. J Clin Endocrinol Metab 92:2816-2820

Neumann S, Claus M, Paschke R (2005) Interactions between the extracellular domain and the extracellular loops as well as the 6th transmembrane domain is necessary for TSH receptor activation. Eur J Endocrinol 152:625-634

Nishi S, Hsu SY, Zell K, Hsueh AJ (2000) Characterization of two fly LGR (leucine-rich repeat-containing, $G$ protein-coupled receptor) proteins homologous to vertebrate glycoprotein hormone receptors: constitutive activation of wild-type flies LGR1 but not LGR2 in transfected mammalian cells. Endocrinology 141:4081-4090

Park SM, Chatterjee VK (2005) Genetics of congenital hypothyroidism. J Med Genet 42:379-389

Parma J, Duprez L, Van Sande J, Cochaux P, Gervy C, Mockel J, Dumont J, Vassart G (1993) Somatic mutations in the thyrotropin receptor gene cause hyperfunctioning thyroid adenomas. Nature 365:649-651

Smits G, Govaerts C, Nubourgh I, Pardo L, Vassart G, Costagliola S (2002) Lysine 183 and glutamic acid 157 of the TSH receptor: two interacting residues with a key role in determining specificity toward TSH and human CG. Mol Endocrinol 16:722-735

Van Sande J, Parma J, Tonacchera M, Swillens S, Dumont J, Vassart G (1995) Somatic and germline mutations of the TSH receptor gene in thyroid diseases. J Clin Endocrinol Metab 80:2577-2585

Yang Y, Guo J, Liu Z, Tang S, Li N, Yang M, Pang Q, Fan F, Bu J, Yuan ST, Xiao X, Chen Y, Zhao K (2006) A locus for autosomal dominant accessory auricular anomaly maps to $14 \mathrm{q} 11.2-\mathrm{q} 12$. Hum Genet 120:144-147 